Commun. math. Phys. 33, 83-96 (1973)

(C) by Springer-Verlag 1973

\title{
Existence, Uniqueness, and Local Stability for the Einstein-Maxwell-Boltzman System
}

\author{
Daniel Bancel* and Yvonne Choquet-Bruhat \\ Département de Mécanique, Université Paris 6, Paris, France
}

Received February 20, 1973

\begin{abstract}
An existence and uniqueness theorem of the solution of the Cauchy problem for the coupled Einstein-Maxwell-Boltzman system is proven, in an appropriate Sobolev space for the potentials, and weighted Sobolev space for the distribution function. The proof relies on a priori estimates for the collision operator previously established by D.B., and for the solution of the Einstein-Maxwell-Liouville system by Y.C.B. It is also proved here that the solution depends continuously on the data.
\end{abstract}

\section{Introduction}

We will, in this paper, give an existence, uniqueness, and local stability theorem for the Einstein-Maxwell-Boltzman system, which rules the dynamics of a relativistic gas, moving under the action of its own gravitationnal and electromagnetic field, the particles of the gas being submitted to binary collisions. Particular cases of such a situation are plasmas in special relativity (the gravitationnal coupling constant has then to be taken zero) or cosmological models of clusters of stars, or clusters of galaxies, which are then the particles of the gas. In the first case the proper masses of the particles take on a finite number of distinct values, in the second case they vary between two positive, finite, numbers. The models considered here are plagued with the usual criticisms about Boltzman equations as representing realistic models. However the Einstein-Maxwell-Liouville system has a very good theoretical basis, for a collisionless relativistic gas, and the Einstein-Maxwell-Boltzman system seems the best correction available to it to take binary encounters into account.

One of us (Y.C.B.) has given previously a proof of existence and uniqueness for the Einstein-Maxwell-Liouville system (cf [1]), the other (D.B.) has proved (cf [2]) an existence and uniqueness theorem for the Boltzman equation in general relativity, using Sobolev spaces which are

* Present address: Département de Mathématiques, Université Paul Sabatier, Toulouse, France. 
also appropriate for the solution of the Einstein-Maxwell equations; such an existence theorem, for the Boltzman equation in a given hyperbolic metric, had been proved previously by Bitcheler [3] by a very elegant method, but the function space in which the solution was found was not adapted to the coupling with other fields, and the general problem of existence for even the Einstein-Boltzman system had not been proved, as was pointed out by Ehlers [4], though a few particular solutions had been constructed [5].

\section{Fundamentals on a Relativistic Kinetic Theory}

We will, in all what follows, restrict ourselves to particles having all the same proper mass $m>0$, the results may be extended to the more general cases quoted in the introduction, with complications in writing.

Phase Space. We denote by $M$ the space time, $C^{\infty}$ manifold endowed with a hyperbolic metric $g$, time oriented. The momenta $p$ of the particles located at a point $x$ of $M$ lie on the mass hyperboloïd:

$$
P_{x}(p): g_{x}(p, p)=g_{\alpha \beta} p^{\alpha} p^{\beta}=m^{2}, \quad p \text { future pointing . }
$$

The phase space $P(M)$ is the fiber bundle with base $M$ and fiber $P_{x}$. The volume element in $P(M)$ is the exterior 7 form $\theta=\eta \wedge \omega$ where $\eta$ is the volume element in $M$

$$
\eta=|g|^{1 / 2} d x^{0} \wedge \cdots \wedge d x^{3}
$$

and $\omega$ the invariant volume element in $P_{x}(p)$

$$
\omega=|g|^{1 / 2} \frac{d p^{1} \wedge d p^{2} \wedge d p^{3}}{p_{0}}
$$

We will always, in taking coordinates, take $x^{i}(i=1,2,3)$ spatial, and $x^{0}$ time like, compatible with the time orientation (i.e. $p^{0}>0$ ).

The trajectory of a particule in $P(M)$ is an orbit of a vector field of the type

$$
X=\left(p^{\alpha}, Q^{\alpha}\right) .
$$

If the only forces acting on the particles are gravitationnal (through the metric $g$ ) and electromagnetic (through an electromagnetic field $F$ ) then ( $e$ charge of the particle)

$$
Q^{\alpha}=-\Gamma_{\lambda \mu}^{\alpha} p^{\lambda} p^{\mu}+e F_{\beta}^{\alpha} p^{\beta} .
$$

In that case, and in all cases where $\left(Q^{\alpha}+\Gamma_{\lambda \mu}^{\alpha} p^{\lambda} p^{\mu}\right) p_{\alpha}=0$, the trajectories lie on $P(M)$, it is well known (Liouville Theorem), and easy to prove, that, under the same hypothesies on $Q^{\alpha}, \theta$ is invariant under $X$ :

$$
\mathscr{L}_{X} \theta=0 \text {. }
$$


Distribution Function. We remark that $\left(i_{X}\right.$ inner product with $X$ )

$$
\theta=\tau \wedge i_{X} \theta
$$

where $\tau$ is a 1-form giving the measure of proper time (if $m$ is normalized to unity) on the trajectories of $X$. The distribution function $f(x, p)$ is a function on $P(M)$, the physical interpretation of which is (cf. also Bel [6]) that $f \theta$ is the measure element for the mean (in the sense of Gibbs ensemble) presence number of particles in $P(M)$; the presence number in a region being the sum of propertimes in which each trajectory of particle remains in the region. Then $i_{X}(f \theta)$ is, as usual, the measure element for the mean number of trajectories crossing a 6-submanifold.

Boltzman Equation. In a collisionless model the physical law of conservation of particles imposes to $f \theta$ to be invariant by the vector field $X$, which, due to (1), gives the Liouville equation

$$
\mathscr{L}_{X} f=p^{\alpha} \frac{\partial f}{\partial x^{\alpha}}+Q^{\alpha} \frac{\partial f}{\partial p^{\alpha}}=0
$$

If collisions are present the zero in the second member, expressing conservation, has to be replaced by a functionnal $\mathscr{I}(f)$, which expresses change in $f$ due to collisions. If these are only binary and elastic it has been shown by Lichnerowricz and Marrot in special relativity [7] and Chernikov in general relativity that

$$
\begin{aligned}
(\mathscr{I}(f))(x, p)= & \int_{P_{x}(q)} \int_{\Sigma_{p, q}}\left\{f\left(x, p^{\prime}\right) f\left(x, q^{\prime}\right)-f(x, p) f(x, q)\right\} \\
& \cdot A\left(x, p, q, p^{\prime}, q^{\prime}\right) \xi^{\prime} \wedge w_{q}
\end{aligned}
$$

where $\Sigma_{p, q}$ is the surface in $P_{x}\left(q^{\prime}\right) \times P_{x}\left(p^{\prime}\right)$ with equation $p^{\prime}+q^{\prime}=p+q$, $\xi^{\prime}$ is the volume element on $\Sigma_{p, q}$, such that $\xi^{\prime} \bigwedge_{\alpha}\left(d p^{\prime \alpha}+d q^{\prime \alpha}\right)=\omega_{p^{\prime}} \wedge \omega_{q^{\prime}}$ for almost all $(p, q)$, in fact for all $(p, q)$ such that $p \neq q$ (or, equivalently, $\left.p^{\alpha} q_{\alpha} \neq m^{2}\right) ; \Sigma_{p, q}$ is a smooth 2-manifold and $\xi^{\prime}$ a smooth 2-form on $\Sigma_{p, q}$ (cf. Ref. $[4,7,8]$, for instance and appendix). $A\left(x, p, q, p^{\prime}, q^{\prime}\right)$ is the cross section for the collisions ( $p, q$ are the momenta of the two particles, at $x$, before collision and $p^{\prime}, q^{\prime}$ their momenta after the collision).

\section{Einstein-Maxwell-Boltzman (E.M.B.) System}

This system, intrinsic on $P(M)$ is constitued with the Boltzman equation

$$
\mathscr{L}_{X} f \equiv p^{\alpha} \frac{\partial f}{\partial x^{\alpha}}-\left(\Gamma_{\lambda \mu}^{\alpha} p^{\lambda} p^{\mu}-e F_{\beta}^{\alpha} p^{\beta}\right) \frac{\partial f}{\partial p^{\beta}}=\mathscr{I}(f)
$$


and the Einstein and Maxwell equations with sources generated by the distribution function $f$

$$
S^{\alpha \beta}=k\left(T^{\alpha \beta}+\tau^{\alpha \beta}\right), \quad T^{\alpha \beta}(x)=\int_{P_{x}} f(x, p) p^{\alpha} p^{\beta} \omega
$$

$\left(\tau^{\alpha \beta}\right.$ is the Maxwell tensor)

$$
\begin{gathered}
\nabla_{\alpha} F^{\alpha \beta}=J^{\beta}, \quad J^{\beta}=e \int_{P_{x}} f(x, p) p^{\beta} \omega, \\
\int_{\alpha, \beta, \gamma} \nabla_{\gamma} F_{\alpha \beta}=0 .
\end{gathered}
$$

In harmonic coordinates, for a vector potential $\varphi^{\alpha}\left[F_{\alpha \beta}=\nabla_{\alpha} \varphi_{\beta}-\nabla_{\beta} \varphi_{\alpha}\right.$, local general solution of (4.b)] normalized by the Lorentz condition, the first members of (3) and (4-a) are

$$
\begin{aligned}
S_{h}^{\alpha \beta} & \equiv-\frac{1}{2} g^{\lambda \mu} \partial_{\lambda \mu}^{2} g^{\alpha \beta}+H^{\alpha \beta}\left(g^{\lambda \mu}, \partial_{\gamma} g^{\lambda \mu}\right) \\
\nabla_{\alpha} F^{\alpha \beta} & \equiv g^{\lambda \mu} \partial_{\lambda \mu}^{2} \varphi^{\beta}+P_{\sigma}^{\beta}\left(g^{\lambda \mu}, \partial_{\gamma} g^{\lambda \mu}\right) \partial_{\varrho} \varphi^{\sigma}
\end{aligned}
$$

standard methods lead from local existence and uniqueness in such gauge conditions to intrinsic and global results (cf. [9]), due to the fact that if $f$ satisfies (2), the corresponding $T^{\alpha \beta}$ and $J^{\beta}$ are conservative $\left(\nabla_{\alpha} T^{\alpha \beta}=0, \nabla_{\beta} J^{\beta}=0\right)$.

Equation (2) is in fact in 7 variables. We will take coordinates in $M$ such that $g^{00}>0, g_{00}>0$, a $\sum_{i=1}^{3}\left(X_{i}\right)^{2} \leqq g^{i j} X_{i} X_{j} \leqq b \sum_{i=1}^{3}\left(X_{i}\right)^{2}$ (with $a$ and $b$ positive numbers) and take, as coordinates on $P_{x}$ the $p^{i}$ s: in these coordinates the image of $P_{x}$ is $\mathbb{R}^{3}, p^{0}$ and $p_{0}$ are $C^{\infty}$ functions of the $p^{i}$ s (if $m \neq 0$ ) depending on $x$ through $g$. It could be predicted and easily verified, that if we still denote by $f$ the distribution function expressed in the variables $\left(x^{\alpha}, p^{i}\right)$, we have:

$$
\mathscr{L}_{X} f \equiv p^{\alpha} \frac{\partial f}{\partial x^{\alpha}}-\left(\Gamma_{\lambda \mu}^{i} p^{\lambda} p^{\mu}-e F_{\beta}^{i} p^{\beta}\right) \frac{\partial f}{\partial p^{i}} .
$$

\section{Local Theorems (Existence, Uniqueness, and Local Stability)}

Definitions. We denote by $\omega_{0}$ a regular ${ }^{1}$ domain of $\mathbb{R}^{3}\left(x^{0}=0\right.$ in $\left.\mathbb{R}^{4}\right)$ and set $\hat{\omega}_{0}=\omega_{0} \times \mathbb{R}^{3}$. The space $H_{\mu}\left(\omega_{0}\right)$ is the Sobolev space of functions having generalized derivatives, up to order $\mu$, square integrable on $\omega_{0}$. The norm in $H_{\mu}\left(\omega_{0}\right)$ is

$$
\|u\|_{H_{\mu}\left(\omega_{0}\right)}^{2}=\sum_{|j| \leqq \mu}\left\|D^{j} u\right\|_{L^{2}\left(\omega_{0}\right)}^{2} .
$$

\footnotetext{
${ }^{1}$ I.e. bounded with lipschitzian boundary, or the whole space.
} 
We denote by $H_{\mu, N}\left(\hat{\omega}_{0}\right)$ the Hilbert space of functions on $\hat{\omega}_{0},\left(x^{i}, p^{i}\right)$ $\rightarrow h\left(x^{i}, p^{i}\right)$, such that the products $\varrho^{N / 2+|\hat{j}|} D^{j} h$ are square integrable, for $|j| \leqq \mu ;|\hat{j}|$ is the number of derivatives in $D^{j}$ which are taken with respect to the $p^{i}$ s;

$$
\varrho^{2}=\sum_{i=1}^{3}\left(p^{i}\right)^{2} .
$$

The norm in $H_{\mu, N}\left(\hat{\omega}_{0}\right)$ is

$$
\|h\|_{H_{\mu, N}}^{2}\left(\hat{\omega}_{0}\right)=\sum_{|j| \leqq \mu}\left\|\varrho^{N / 2+|\hat{j}|} D^{j} h\right\|_{L^{2}\left(\omega_{0}\right)} .
$$

Analogously if $\Omega$ is a domain of $\mathbb{R}^{4}, H_{\mu}(\Omega)$ is the usual Sobolev space of functions on $\Omega$, with norm

$$
\|u\|_{H_{\mu}(\Omega)}^{2}=\sum_{|j| \leqq \mu}\left\|D^{j} u\right\|_{L^{2}(\Omega)}^{2},
$$

and we denote by $H_{\mu, N}(\hat{\Omega}), \hat{\Omega}=\Omega \times \mathbb{R}^{3}$, the Hilbert space of functions on $\hat{\Omega},\left(x^{\alpha}, p^{i}\right) \rightarrow f\left(x^{\alpha}, p^{i}\right)$, with norm

$$
\|f\|_{H_{\mu, N}(\hat{\Omega})}=\sum_{|j| \leqq \mu}\left\|\varrho^{N / 2+|\hat{j}|} D^{j} f\right\|_{L^{2}(\hat{\Omega})} .
$$

In all these definitions we will accept the value $N=+\infty$, being understood that, here

$$
\varrho^{\infty}=e^{\varrho} .
$$

Note. if $g^{\alpha \beta}$ is an uniformly hyperbolie metric on $\Omega$, with $x^{0}=C^{t e}$ regularly spatial (i.e. $g^{00} \geqq a>0, g^{i j} X_{i} X_{j}$ negative definite), there exists two positive constants $A$ and $B$ such that, on $\hat{\Omega}$, for all strictly time like vector field $v$ (and in particular for $v_{\lambda}=\delta_{\lambda}^{0}$ )

if

$$
A v_{\lambda} p^{\lambda} \leqq \varrho \leqq B v_{\lambda} p^{\lambda}
$$

$$
g_{\alpha \beta} p^{\alpha} p^{\beta}=m^{2}
$$

the norm $(2-1)$ is thus equivalent to

$$
\sum_{|j| \leqq \mu}\left\|\left(v_{\lambda} p^{\lambda}\right)^{N / 2+|\hat{j}|} D^{j} f\right\|_{L^{2}(\hat{\Omega})} .
$$

We will say that a cross section $A$ is $\mu-N$ regular in $\hat{\Omega}$ if $\frac{1}{p^{0}} \mathscr{I}(f)$ is a bounded quadratic form in the space $H_{\mu, N}(\hat{\Omega})$, or, more precisely, if there exists a positive number I such that, for each $\hat{\omega}_{t}=\hat{\Omega} \cap\left\{x^{0}=t\right\}$ the following inequality is satisfied

$$
\left\|\frac{1}{p^{0}} \mathscr{I}(f)\right\|_{H_{\mu, N}\left(\hat{\omega}_{t}\right)} \leqq I\|f\|_{H_{\mu}, N}^{2}\left(\hat{\omega}_{t}\right) .
$$


We will study, in the appendix, the condition under which a cross section is regular.

Cauchy Datas. We give, on $\omega_{0}, 28$ functions

such that

$$
\begin{gathered}
\gamma^{\alpha \beta}, \psi^{\alpha} \in H_{\mu+1}\left(\omega_{0}\right) ; \quad \gamma^{\prime \alpha \beta}, \psi^{\prime \alpha} \in H_{\mu}\left(\omega_{0}\right) \\
\left|\eta^{\alpha \beta}-\gamma^{\alpha \beta}\right| \leqq \varepsilon-\delta, \delta>0, \quad \alpha, \beta=0,1,2,3
\end{gathered}
$$

where $\varepsilon$ is such that, for all $\delta \geqq 0$ the inequality (2.2) insures the uniform hyperbolicity of $\gamma$ and spatiality of $\omega_{0}$ with respect to $\gamma$ : due to the local character of our present study the hypothesis (2.3) is not a restriction on the geometric Cauchy data. We also give, on $\hat{\omega}_{0}$, a function

$$
h \in H_{\mu, N}\left(\hat{\omega}_{0}\right) .
$$

We suppose that, in a neighborhood $\hat{\omega}_{0} \times[-T, T]$ of $\hat{\omega}_{0}$, the cross section $A$ is regular.

We will then prove the following:

Theorem. There exist, if $\mu \geqq 5, N \geqq 6$, a domain $\Omega$ in $\mathbb{R}^{4}$ (which may be chosen globally hyperbolie for the constructed metric $g^{\alpha \beta}$, and admitting $\omega_{0}$ as a Cauchy surface), a metric $g^{\alpha \beta}$ and a vector $\varphi^{\alpha}$ on $\Omega$, and a function $f$ on $\hat{\Omega}=\Omega \times \mathbb{R}^{3}$ such that

1) $\varphi^{\alpha}, g^{\alpha \beta} \in H_{\mu+1}(\Omega), f \in H_{\mu, N}(\hat{\Omega})$

2) $g^{\alpha \beta}, \varphi^{\alpha}, f$ satisfy the E.M.B. system

3) $g^{\alpha \beta}, \varphi^{\alpha}$ and $f$ take on $\omega_{0}$, and $\hat{\omega}_{0}$, the Cauchy data (2.2), (2.4):

$$
\begin{gathered}
g^{\alpha \beta} / \omega_{0}=\gamma^{\alpha \beta}, \quad \partial_{0} g^{\alpha \beta} / \omega_{0}=\gamma^{\prime \alpha \beta}, \quad \varphi^{\alpha} / \omega_{0}=\psi^{\alpha}, \quad \partial_{0} \varphi^{\alpha}=\psi^{\prime \alpha}, \\
f / \hat{\omega}_{0}=h .
\end{gathered}
$$

This solution is unique in $\Omega$ and depends continuously on the data.

Note. The existence domain $\Omega$ depends only on the norms of the Cauchy data, and, in particular, is just translated by a translation of $\omega_{0}$ in $\mathbb{R}^{3}$, this result insures the "globality in space" of the solution, without shrinking to zero at infinity, without any need of minkovskian asymptotic behaviour.

Proof. It relies on the energy inequalities and contracting mapping principle.

Let us denote by $U$ a domain of $\mathbb{R}^{4}$, globally hyperbolic and admitting $\omega_{0}$ as a Cauchy surface for all $C^{0}$ metrics such that, on $U$

$$
\sup _{\alpha, \beta}\left|g^{\alpha \beta}-\eta^{\alpha \beta}\right| \leqq \varepsilon .
$$

We will denote by $\Omega_{T}$ the strip

$$
\Omega_{T}=U \cap\left\{0 \leqq x^{0} \leqq T\right\}
$$


and set, for a metric $g$ on $\Omega_{T}$ :

$$
\|g-\eta\|_{H_{\mu+1}\left(\Omega_{T}\right)}=\sup _{\alpha, \beta}\left\|g^{\alpha \beta}-\eta^{\alpha \beta}\right\|_{H_{\mu+1}\left(\Omega_{T}\right)} .
$$

With the help of Sobolev inclusion theorem it can be proved (cf. [1]) that there exists a positive constant $M$, independant of $T$, such that, if $\mu \geqq 3,\left(g\right.$ is then $\left.C^{1}\right)$ and if $g$ takes on $\omega_{0}$ values satisfying (2.3), the inequality

implies (2.5).

$$
\|g-\eta\|_{H_{\mu+1}}\left(\Omega_{T}\right) \leqq M
$$

Let us then denote by $\stackrel{1}{g}$ a metric on $\Omega_{T}$ satisfying (2.6a) taking on $\Omega_{0}$ the given values and by $\varphi$ a vector potential on $\Omega_{T}$ such that

$$
\|\|_{\varphi}^{1}\left\|_{H_{\mu+1}\left(\Omega_{T}\right)}=\sup _{\alpha}\right\| \hat{\varphi}^{\alpha} \|_{H_{\mu+1}\left(\Omega_{T}\right)} \leqq K
$$

where $K$ is an arbitrary given constant.

We denote by $\stackrel{1}{f}$ the distribution function solution of the Cauchy problem

$$
\stackrel{1}{f} \mid \omega_{0}=h
$$

for the Boltzman equation relative to $\stackrel{1}{g}$ :

$$
\hat{p}^{\alpha} \frac{\partial \stackrel{1}{f}^{\alpha}}{\partial x^{\alpha}}-\stackrel{1}{Q}^{i} \frac{\partial f^{1}}{\partial p^{i}}=\mathscr{I}(\stackrel{1}{f}) \text {. }
$$

We know that for a $\mu-N$ regular cross section and if $\mu \geqq 5$, (cf. [2]) there exists numbers $T_{1}, 0<T_{1} \leqq T$ and $K_{1}>0$, depending only on $M, K,\|h\|_{H_{\mu, N}\left(\hat{\omega}_{0}\right)}$, such that

$$
\left\|f^{1}\right\|_{H_{\mu, N}\left(\hat{\Omega}_{T_{l}}\right)}<K_{1} .
$$

We then consider the hyperbolic linear system, with unknowns $\stackrel{2}{g}, \stackrel{2}{\varphi}$

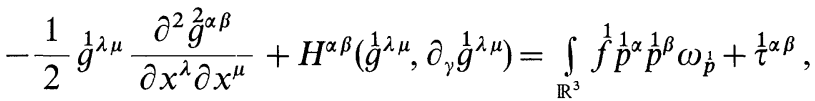

$$
\begin{aligned}
& \stackrel{1}{g}^{\lambda \mu} \frac{\partial^{2} \underline{\varphi}^{\beta}}{\partial x^{\lambda} \partial x^{\mu}}+P_{\sigma}^{\beta \varrho}\left(\frac{1}{g}{ }^{\lambda \mu}, \partial_{\gamma}{ }^{\lambda}{ }^{\lambda \mu}\right) \partial_{\varrho}{ }^{2} \varphi^{\sigma}=e \int_{\mathbb{R}^{3}} \stackrel{1}{f}^{1}{ }^{1} \omega_{p}^{1},
\end{aligned}
$$

the coefficients of the first members are in $H_{\mu}\left(\Omega_{T_{1}}\right)$ (recall that if $\mu \geqq 3$, $H_{\mu}\left(\Omega_{T_{1}}\right)$ is an algebra) and the same is true for the second members if $N \geqq 6$, due to 2.7 (cf. [1]); we have:

$$
\begin{aligned}
& \left(T^{\alpha \beta}\right)^{2}=\left(\int_{\mathbb{R}^{3}} f^{1} p^{\alpha} p^{\beta} \omega_{\hat{p}}\right)^{2} \leqq \int_{\mathbb{R}^{3}} \varrho^{N}\left(\frac{1}{f}\right)^{2}\left|\frac{1}{g}\right|^{1 / 2} d p^{1} d p^{2} d p^{3} \\
& \cdot \int_{\mathbb{R}^{3}} \varrho^{-N}\left(\frac{p^{\alpha} p^{\beta}}{p_{0}}\right)^{2}|g|^{1 / 2} d p^{1} d p^{2} d p^{3}
\end{aligned}
$$


the second integral on $\mathbb{R}^{3}$ is bounded by a number $C$, depending only on $\varepsilon$ (i.e. on $M$ ), if $N>5$. Therefore

$$
\left\|T^{1 / \beta}\right\|_{L^{2}\left(\Omega_{T_{l}}\right)}^{2} \leqq C\left\|f^{1}\right\|_{H_{0, N}\left(\hat{\Omega}_{T_{l}}\right)}^{2}
$$

a straight foward calculation (see analogous in [1]) shows also that, there exist $C$, depending only on $M$, such that:

$$
\left\|\stackrel{1}{T}^{\alpha \beta}\right\|_{H_{\mu}\left(\Omega_{T_{l}}\right)}^{2} \leqq C \| \stackrel{1}{f}_{H_{\mu, N}\left(\hat{\Omega}_{T_{l}}\right)}^{2}
$$

(in fact a stronger inequality where derivatives of $f^{1}$ with respect to $p$ do not appear is also valid).

We can therefore apply the Leray-theorem about hyperbolic linear equations with coefficients in $H_{\mu}\left(\Omega_{T_{1}}\right)$ to (2.8): the Cauchy problem (2.2) relative to this system, has one and only one solution $\stackrel{2}{g}, \stackrel{2}{\varphi}$ in $\left(\times H_{\mu+1}\left(\Omega_{T_{1}}\right)\right)^{14}$, and there exists a number $T_{2}, 0<T_{2} \leqq T_{1}$ such that

$$
\left.\|\stackrel{2}{g}-\eta\|_{H_{\mu+1}\left(\Omega_{T_{2}}\right.}\right)<M, \quad\|\stackrel{2}{\varphi}\|_{H_{\mu+1}\left(\Omega_{T_{2}}\right)}<K .
$$

If we restrict $\stackrel{1}{g}$ and $\stackrel{1}{\varphi}$ to $\Omega_{T_{2}}$, the solution of the Cauchy problem for (2.8) defines therefore a mapping $\mathscr{F}:(\stackrel{1}{g}, \stackrel{1}{\varphi}) \rightarrow(\stackrel{2}{g}, \stackrel{2}{\varphi})$ from the ball $B$ in $\left(\times H_{\mu+1}\left(\Omega_{T_{2}}\right)\right)^{14}$ :

$$
B:\|g-\eta\|_{H_{\mu+1}\left(\Omega_{T_{2}}\right)} \leqq M, \quad \cdot\|\varphi\|_{H_{\mu+1}\left(\Omega_{T_{2}}\right)} \leqq K
$$

into itself.

To show that the mapping $\mathscr{F}$ is continous we set if $(\stackrel{1}{g}, \stackrel{1}{\varphi})$ and $\left(\stackrel{1}{g}^{\prime}, \underline{\varphi}^{\prime}\right)$ are two points of $B$ :

and also

$$
\stackrel{1}{G}=\stackrel{1}{g}^{\prime}-\stackrel{1}{g} \quad \stackrel{1}{\Phi}=\stackrel{1}{\varphi^{\prime}}-\stackrel{1}{\varphi}
$$

$$
\stackrel{2}{G}=\stackrel{2}{g}^{\prime}-\stackrel{2}{g} \quad \stackrel{2}{\Phi}=\stackrel{2}{\varphi}^{\prime}-\stackrel{2}{\varphi} \text {. }
$$

By substracting the partial differential equations satisfied by $\stackrel{2}{g}$ and $\stackrel{2}{g}$ we find:

$$
\begin{gathered}
\frac{1}{2} \stackrel{1, \alpha \beta}{g^{\prime \alpha}} \frac{\partial^{2}}{\partial x^{\alpha} \partial x^{\beta}} \stackrel{2}{G}^{\lambda \mu}+\frac{1}{2} \stackrel{1}{G^{\alpha \beta}} \frac{\partial^{2}}{\partial x^{\alpha} \partial x^{\beta}} \stackrel{2}{g}^{\lambda \mu}+\stackrel{1}{H}^{\alpha \beta}-\stackrel{1}{H}^{\alpha \beta \beta} \\
=T^{\alpha \beta}-\stackrel{1}{T}^{\prime \alpha \beta}+\stackrel{1}{\tau}^{\alpha \beta}-{ }^{1 / \alpha \beta}
\end{gathered}
$$

and analogous equations for $\stackrel{2}{\Phi}$ by substracting the corresponding equations for $\stackrel{2}{\varphi}$ and $\stackrel{2}{\varphi}^{\prime}$.

The Leray theory for hyperbolic linear equations (energy inequalities after $\mu-1$ derivations) gives, as in [1] that $(\stackrel{2}{G}, \stackrel{2}{\Phi})$ tends to zero in 
$\left(\times H_{\mu}\left(\Omega_{T_{2}}\right)\right)^{14}$ when it is so for $(\stackrel{1}{G}, \stackrel{1}{\Phi})$. The mapping $\mathscr{F}$ is thus a continous mapping from the ball $B$, convex compact set, of $\left(\times H_{\mu}\left(\Omega_{T_{2}}\right)\right)^{14}$, into it self, it has, by Schauder theorem, a fixed point - which is the required solution of the considered Cauchy problem.

The uniqueness of the solution can be proved directly: it is a simple consequence of the energy inequalities.

\section{Appendix}

Parameters on $\Sigma_{p, q}$. When $p \neq q$ (or, equivalent $p_{\alpha} q^{\alpha} \neq m^{2}$ ), $\Sigma_{p, q}$ is a smooth 2 - surface in $P_{x}\left(p^{\prime}\right) \times P_{x}\left(q^{\prime}\right)$.

Let us set

$$
p+q=2 \lambda \tilde{e}_{0}
$$

where $\lambda$ is a positive number, and $\tilde{e}_{0}$ a vector of length 1 in the metric $g$ at $x$.

$\Sigma_{p, q}$ being defined by

$$
p^{\prime}+q^{\prime}=p+q=2 \lambda \tilde{e}_{0}
$$

we have, since $p, q, p^{\prime}, q^{\prime}$ have length $m$ :

$$
p^{\alpha} q_{\alpha}=p^{\prime \alpha} q_{\alpha}^{\prime}=2 \lambda^{2}-m^{2} \geqq m^{2}
$$

and $p^{\prime \alpha} q_{\alpha}^{\prime}=p^{\alpha} q_{\alpha}=m^{2}$ only if $p=q$ and then also $p^{\prime}=q^{\prime}$ (and $\lambda=m$ ).

In an orthonormal frame with time axis $\tilde{e}_{0}$ we have

therefore

$$
\tilde{p}^{\prime 0}+\tilde{q}^{\prime 0}=2 \lambda, \quad \tilde{p}^{\prime i}+\tilde{q}^{\prime i}=0
$$

hence

$$
p^{\prime \alpha} q_{\alpha}^{\prime}=\tilde{p}^{\prime 0}\left(2 \lambda-\tilde{p}^{\prime 0}\right)+\sum_{i=1}^{3}\left(\tilde{p}^{\prime i}\right)^{2}=2 \lambda^{2}-m^{2}
$$

$$
\tilde{p}^{\prime 0}=\lambda, \quad \sum_{i=1}^{3}\left(\tilde{p}^{\prime i}\right)^{2}=\lambda^{2}-m^{2} .
$$

In this frame $\Sigma_{p, q}$ is a $2-$ sphere of the plane $\tilde{p}^{\prime}=\lambda$. Let us take as axis for polar coordinates $\theta, \varphi$ on $\Sigma_{p, q}$ a vector parallele to $p-q$ (which is orthogonal to $p+q$ ), $\theta$ is the angle of $p-q$ with $p^{\prime}-q^{\prime}$ and set, on $\Sigma_{p, q}$

with

$$
\tilde{p}^{\prime 1}=\alpha \cos \theta, \quad \tilde{p}^{\prime 2}=\alpha \sin \theta \cos \varphi, \quad \tilde{p}^{\prime 3}=\alpha \sin \theta \sin \varphi
$$

$$
\alpha=\left(\lambda^{2}-m^{2}\right)^{1 / 2}=\left[\frac{1}{2}\left(p^{\alpha} q_{\alpha}-m^{2}\right)\right]^{1 / 2}=\left[-\frac{1}{4}\left(p^{\alpha}-q^{\alpha}\right)\left(p_{\alpha}-q_{\alpha}\right)\right]^{1 / 2}=\frac{1}{2}|p-q|
$$


In these coordinates $\theta, \varphi$ the volume element $\xi^{\prime}$ on $\Sigma_{p, q}$ is

If we set

$$
\xi^{\prime}=\frac{\alpha}{2 \lambda} \sin \theta d \theta \wedge d \varphi
$$

$$
S(x, p, q, \theta, \varphi)=A \alpha / 2 \lambda .
$$

the Boltzman equation reads

$$
\begin{gathered}
\mathscr{L}_{X}(f)=\mathscr{I}(f) \int_{P_{x}(q)} \int_{0}^{\pi} \int_{0}^{2 \pi}\left\{f\left(x, p^{\prime}\right) f\left(x, q^{\prime}\right)-f(x, p) f(x, q)\right\} \\
\cdot S(x, p, q, \theta, \varphi) \sin \theta d \theta \wedge d \varphi \wedge \omega_{q} .
\end{gathered}
$$

\section{Energy Estimates}

The energy estimates are obtained by multiplying the $D^{l}$-derivative of both members of (1) by $D^{l} f \cdot h_{l}$ with $h_{l}(p)=\left(v_{\lambda} p^{\lambda}\right)^{N+2|\hat{l}|}$.

We have if, $l=0, h=h_{0}$

$$
f h \mathscr{L}_{X} f=\frac{1}{2} \mathscr{L}_{X}\left(h f^{2}\right)-\frac{1}{2} f^{2} \mathscr{L}_{X} h
$$

which gives, by integration on a tube $\hat{\Omega}_{T}$ in phase space with lateral boundaries generated by particle paths (cf. [1], $\hat{\Omega}_{T}=\Omega_{T} \times \mathbb{R}^{3}, \Omega_{T}$ domain of $\mathbb{R}^{4}$ )

$$
\frac{1}{2} \int_{\partial \mathscr{\Omega}_{T}} h f^{2} i_{X} \theta-\frac{1}{2} \int_{\hat{\Omega}_{T}} f^{2} \mathscr{L}_{X} h \theta=\int_{\hat{\Omega}_{T}} f \cdot h \cdot \mathscr{I}(f) \theta .
$$

In the case $N=+\infty$ one uses Bitcheler's lemma [3] (existence of a time like vector field $v$ such that $\mathscr{L}_{X}\left(v_{\lambda} p^{\lambda}\right) \leqq 0$ ) extended to all regular metrics on $\Omega_{T}$, to obtain the estimate $\left(\omega_{t}=\Omega_{T} \cap x^{0}=t, \hat{\omega}_{t}=\omega_{t} \times \mathbb{R}^{3}\right)$,

$$
\begin{aligned}
\int_{\omega_{t}} h f^{2} p^{0}|g|^{1 / 2} d^{3} x \wedge \omega_{p} \leqq \int_{\omega_{0}} h f^{2} p^{0}|g|^{1 / 2} d^{3} x \wedge \omega_{p} \\
=\int_{\Omega_{t}} f^{2} h|g|^{1 / 2} d^{3} p \wedge \eta+\int_{\Omega_{t}} h\left(p_{0}\right)^{-2}|\mathscr{I}(f)|^{2} d^{3} p \wedge \eta .
\end{aligned}
$$

In the case $N$ finite the identity

$$
\mathscr{L}_{X} h=\mathscr{L}_{X}\left[\left(v_{\lambda} p^{\lambda}\right)^{N}\right]=N\left(v_{\lambda} p^{\lambda}\right)^{N-1} p^{\alpha} p^{\beta} \nabla_{\alpha} p_{\beta}
$$

enables us to obtain an analogous estimate with some constant $C$ in front of the second integral of the second member, without use of Bitcheler's lemma (cf. [1]).

In all cases one uses the fact that, in phase-space, $i_{X} \theta$ vanishes on the lateral boundary of $\Omega_{T}$, and that the part $\omega_{0}^{\prime}$ of this boundary corresponding to $x^{0}=0$ is included in $\hat{\omega}_{0}=\omega_{0} \times \mathbb{R}^{3}$. 


\section{$\mu-N$ Regular Cross-Section}

The mathematical asumption on the operator $\mathscr{I}$ is the existence of a constant I such that

We write

$$
\left\|\frac{1}{p^{0}} \mathscr{I}(f)\right\|_{H_{\mu, N}\left(\omega_{t}\right)} \leqq I\|f\|_{H_{\mu, N}\left(\hat{\omega}_{t}\right)}^{2} .
$$

with

$$
\mathscr{I}(f)=\mathscr{I}_{2}(f)-\mathscr{I}_{1}(f)
$$

$$
\begin{aligned}
\mathscr{I}_{1}(f)= & \int_{P_{x}(q)} \int_{0}^{\pi} \int_{0}^{2 \pi} f(x, p) f(x, q) S(x, p, q, \theta, \varphi) \\
& \cdot \sin \theta d \theta \wedge d \varphi \wedge \omega_{q} \\
\mathscr{I}_{2}(f)= & \int_{P_{x}(q)} \int_{0}^{\pi} \int_{0}^{2 \pi} f\left(x, p^{\prime}\right) f\left(x, q^{\prime}\right) S(x, p, q, \theta, \varphi) \\
& \cdot \sin \theta d \theta \wedge d \varphi \wedge \omega_{q} .
\end{aligned}
$$

For $\mu=0, N \leqq+\infty$ we have

$$
\begin{aligned}
\left|\mathscr{I}_{1}(f)\right|^{2} \leqq & |f(x, p)|^{2} \cdot 2 \pi \cdot \int|f(x, q)|^{2} h(q) d^{3} q \\
& \cdot \int h^{-1}(q) S^{2}\left(q_{0}\right)^{-1} \sin \theta d \theta \wedge d \varphi \wedge \omega_{q}
\end{aligned}
$$

if we suppose, $C$ being a positive number

$$
\int_{P_{x}(q)} \int_{0}^{\pi} \int_{0}^{2 \pi} h^{-1}(q)\left(q_{0}\right)^{-1} S^{2} \sin \theta d \theta \wedge d \varphi \wedge \omega_{q} \leqq C\left(p^{0}\right)^{2}
$$

we have using Sobolev inequalities (cf. [2])

$$
\left\|\frac{1}{p^{0}} \mathscr{I}_{1}(f)\right\|_{H_{0, N}\left(\hat{\omega}_{t}\right)} \leqq I\|f\|_{H_{\mu, N}\left(\hat{\omega}_{t}\right)}^{2} \quad \text { if } \quad \mu \geqq 5
$$

where $I$ is a constant depending on $\Omega$ and $\mu$. We have the estimate (2) for $D^{l}$ derivative of $\frac{1}{p^{0}} \mathscr{I}_{1}(f)$

if we suppose, in the case $N$ finite,

$\sum_{|l+k| \leqq \mu}\left(p^{0}\right)^{2\lceil|l+k|} \int h_{l}^{-1}(q)\left(q^{0}\right)^{-1}\left|D^{k} S\right|^{2} \sin \theta d \theta \wedge d \varphi \wedge \omega_{q} \leqq C\left(p^{0}\right)^{2}$

and in the case $N=+\infty$

$$
\sum_{|l| \leqq \mu} \int h(-q)\left(q^{0}\right)^{-1}\left|D^{l} S\right|^{2} \sin \theta d \theta \wedge d \varphi \wedge \omega_{q} \leqq C\left(p^{0}\right)^{2} .
$$

On the other hand we have, for $\mu=0$,

$$
\begin{aligned}
\left|\mathscr{I}_{2}(f)\right|^{2} \leqq & \int\left|f\left(x, p^{\prime}\right)\right|^{2} h\left(p^{\prime}\right)\left(p_{0}^{\prime}\right)\left|f\left(x, q^{\prime}\right)\right|^{2} h\left(q^{\prime}\right) \frac{q_{0}^{\prime}}{p_{0}} h^{-1}(p) \xi \wedge \omega_{q} \\
& \cdot \int S^{2} \frac{h^{-1}\left(p^{\prime}\right)}{p_{0}^{\prime}} \cdot \frac{h^{-1}\left(q^{\prime}\right)}{q_{0}^{\prime}} \cdot p_{0} \cdot h(p) \sin \theta d \theta \wedge d \varphi \wedge \omega_{q}
\end{aligned}
$$


which will give (using the fact on $\Sigma_{p, q}$ we have

$$
\begin{gathered}
\left.\xi^{\prime} \wedge \omega_{q} \wedge \omega_{p}=\xi \wedge \omega_{q^{\prime}} \wedge \omega_{p^{\prime}}\right) \\
\left\|\frac{1}{p^{0}} \mathscr{I}_{2} f\right\|_{H_{0, N}\left(\omega_{t}\right)} \leqq I\|f\|_{H_{\mu, N}\left(\hat{\omega}_{t}\right)}^{2}
\end{gathered}
$$

if their exist a constant $C$ such that:

$$
\int_{P_{x}(q)} \int_{0}^{\pi} \int_{0}^{\pi} S^{2} \sin \theta d \theta \wedge d \varphi \wedge \omega_{q} \leqq C\left(p^{0}\right)^{2}
$$

(in the case $N=+\infty$, we use the fact that on the surface $\Sigma_{p, q}$, $v_{\lambda}\left(p^{\prime \lambda}+q^{\prime \lambda}-p^{\lambda}\right)=v_{\lambda} q^{\lambda}$, and in the case $N$ finite the inegality $\left.p^{0} \leqq C p^{\prime 0} q^{\prime 0}\right)$. The estimates for $D^{l}$ derivatives of $\frac{1}{p^{0}} \mathscr{I}_{2}(f)$ are more difficult to obtain. Our unknown, from the analyst point of view, is the function $f\left(x^{\alpha}, p^{i}\right)$ [denote by abuse of language $f(x, p)$ ] expression of the distribution function in a given family of frames $\left(x^{\alpha}, e_{\alpha}\right)$ on the manifold $M$ : to compute explicitly $\mathscr{I}_{2}(f)$ we have to express the components in such a frame of $p^{\prime}$ and $q^{\prime}$ in terms of $p, q, \theta, \varphi$. For this purpose we introduce the relative velocity of incident particules in the given frame

$$
\boldsymbol{v}=\frac{\left(e_{0} \cdot q\right) \boldsymbol{p}-\left(e_{0} \cdot p\right) \boldsymbol{q}}{\left(e_{0} \cdot p\right)\left(e_{0} \cdot q\right)}
$$

$e_{0}$ is the time axis, future directed of the given frame of the tangent space $T_{x}, \boldsymbol{p}$ denotes the spatial component of the vector $p$.

Let us set

$$
\tilde{e}_{0}^{\prime}=\frac{p+q}{2 \lambda}, \quad \tilde{e}_{1}^{\prime}=\frac{p-q}{2 \alpha}, \quad \tilde{e}_{2}^{\prime}=\frac{\boldsymbol{\sigma}}{\sigma}, \quad \tilde{e}_{3}^{\prime}=\frac{\sigma}{\lambda \alpha}\left[e_{0}+\frac{p^{0} q^{0}}{\sigma^{2}} \boldsymbol{v} \wedge \boldsymbol{\sigma}\right]
$$

with

We have $\sigma^{2}=\left(p^{0} q^{0}\right)^{2} v^{2}-\alpha^{2} \lambda^{2}, \quad p=p^{0} e_{0}+\boldsymbol{p}, \quad \boldsymbol{\sigma}=\boldsymbol{p} \wedge \boldsymbol{q}$.

if we set

$$
p^{\prime}=\frac{p+q}{2}+\alpha n, \quad q^{\prime}=\frac{p+q}{2}-\alpha n
$$

therefore

$$
n=\cos \theta \tilde{e}_{1}^{\prime}+\sin \theta \cos \varphi \tilde{e}_{2}^{\prime}+\sin \theta \cos \varphi \tilde{e}_{3}^{\prime}
$$

$$
\boldsymbol{p}^{\prime}=\frac{\boldsymbol{p}+\boldsymbol{q}}{2}+\cos \theta \frac{\boldsymbol{p}-\boldsymbol{q}}{2}+\alpha \sin \theta \sin \varphi \frac{\boldsymbol{\sigma}}{\sigma}+\sin \theta \cos \varphi \frac{p^{0} q^{0}}{2 \lambda}\left(\boldsymbol{v} \wedge \frac{\boldsymbol{\sigma}}{\sigma}\right) .
$$

Let us denote by $g^{(v)}$ any linear combination of products $\left(\Pi D^{l} g_{\lambda \mu}\right)$ with $\Sigma|l| \leqq v$, with coefficients in the algebra of bounded function on $\Omega \times \mathbb{R}^{3} \times \mathbb{R}^{3}$ generated by

$$
p^{\alpha} / p^{0}, \quad p^{\alpha} / p^{0}, \quad q^{\alpha} / q^{0}, \ldots
$$


From the formulas

$$
\frac{\partial p^{0}}{\partial x^{\alpha}}=-\frac{p^{\lambda} p^{\mu}\left(\partial g_{\lambda \mu} / \partial x^{\alpha}\right)}{2 p_{0}}, \quad \frac{\partial p^{0}}{\partial p_{i}}=-\frac{p_{i}}{p_{0}}
$$

one deduce

$$
\frac{\partial}{\partial p^{i}}\left[g^{(v)}\right]=\frac{g^{(v)}}{p^{0}} ; \quad \frac{\partial}{\partial x^{\alpha}}\left[g^{(v)}\right]=g^{(v+1)}
$$

thus the estimates, $l=\bar{l}+\hat{l}$,

$$
\begin{aligned}
& D^{l}\left(v^{j}\right)=\left(p^{0}\right)^{-|\hat{\imath}|} g^{(|\bar{l}|)} ; \quad D^{l}(\alpha)=\frac{\left(p^{0}\right)^{|\bar{l}|} g^{|\bar{l}|}}{\alpha^{(2|l|-1)}} . \\
D^{l}\left(\frac{\boldsymbol{\sigma}}{\sigma}\right)=0 \quad \text { if } \quad l \neq \hat{l} & g^{(0)} \frac{\left(q^{0}\right)^{|l|}}{\sigma^{|l|}}-\text { if } \quad l=\hat{l} ; \quad\left|D^{l}\left(\frac{1}{\lambda}\right)\right|=\frac{\left(p^{0}\right)^{|\bar{l}|}\left(q^{0}\right)^{|l|}}{\lambda^{2|l|+1}} g^{(|l|)} .
\end{aligned}
$$

Let us denote

From

$$
\psi_{v}=\sum_{\substack{|r+s| \leqq v \\ r=r_{1}+r_{2}}} \frac{\left(p^{0} q^{0}\right)^{|r|+|\hat{s}|+1 / 2}}{\lambda^{2\left|r_{1}\right|} \alpha^{2\left|r_{2}\right|} \sigma^{|\hat{s}|}}
$$

$$
D^{l}\left(p^{\prime}\right)=\frac{1+\cos \theta}{2} D^{l} \boldsymbol{p}+\sin \theta \cos \varphi D^{l}\left(\frac{\boldsymbol{\sigma}}{\sigma}\right)+\cos \varphi D^{l}\left(p^{0} q^{0}\left(v \wedge \frac{\boldsymbol{\sigma}}{\sigma}\right)\right.
$$

results the estimate

$\left|D^{l} p^{\prime}\right| \leqq g^{(\bar{l})}\left(p^{0}\right)^{-|\hat{l}|} \psi|l|$.

(For example $D^{l}\left(\alpha \frac{\boldsymbol{\sigma}}{\sigma}\right)$ is a linear combination with constant coef-

ficients of terms $D^{r} \alpha D^{s}\left(\frac{\boldsymbol{\sigma}}{\sigma}\right), r+s=l$, which admit the estimate

$$
\frac{g^{(\bar{r})}\left(p^{0}\right)^{|\bar{r}|}\left(q^{0}\right)^{|r|}}{\alpha^{2|r|-1}} \cdot \frac{g^{(0)}\left(q^{0}\right)^{|\hat{s}|}}{\sigma^{|\hat{s}|}} \leqq g^{(r)} \cdot \frac{1}{\left(p^{0}\right)^{|\ell|}} \cdot \frac{\alpha \cdot\left(p^{0} q^{0}\right)^{|r|+|\hat{s}|}}{\sigma^{2|r|} \sigma^{|\hat{s}|}}
$$

Using these estimates, the usual laws of derivation and Schwarz inequality, one gets

$$
\left\|\frac{1}{p^{0}} \mathscr{I}_{2} f\right\|_{H_{\mu, N}\left(\hat{\omega}_{t}\right)} \leqq I\|f\|_{H_{\mu, N}\left(\hat{\omega}_{t}\right)}^{2}, \quad \mu \geqq 5
$$


if, for $N$ finite,

$$
\sum_{|m| \leqq \mu} \int\left|D_{x, p, q}^{m} S\right|^{2} \psi_{|m|}^{2} \sin \theta d \theta \wedge d \varphi \wedge \omega_{q} \leqq C\left(p^{0}\right)^{2}
$$

or if, for $N=+\infty$,

$$
\sum_{|m| \leqq \mu} \int\left|D_{x, p, q}^{m} S\right|^{2} \cdot \psi_{|m|}^{2} \cdot e^{-q^{0}} \cdot \sin \theta d \theta \wedge \vec{d} \varphi \wedge \omega_{q} \leqq C\left(p^{0}\right)^{2} .
$$

\section{References}

1. Choquet-Bruhat, Y.: Problème de Cauchy pour le système intégro-différentiel d'Einstein-Liouville. Ann. Inst. Fournier, XXI, 3, 1971, p.181-201 and Einstein-MaxwellLiouville system, volume in the honour of Petrov

2. Bancel,D.: Problème de Cauchy pour l'équation de Boltzman en relativité générale (to be published, Annales de l'Institut Poincaré)

3. Bitcheler, K.: On the Cauchy problem for relativistic Boltzman equation. Commun. math. Phys. 4, 352-364, 1967

4. Ehlers, J.: General relativity and kinetic theory. In: Proceedings of the Int. School of Physics "Enrico Fermi" Course 47 - p. 1.70 New York: Acad. Press 1971

5. Ehlers, J., Sachs, R.K.: Kinetic theory and cosmology in Astrophysics and General Relativity. New York: Gordon Breach 1971

6. Bel,L.: Kinetic theory of cosmology. Astro physical. J. 55, 83-87 (1969)

7. Lichnerowicz, A., Marrot, R.: Propriétés statistiques des ensembles de particules en relativité restreinte. C.R. 210, 759-61 (1940)

8. Israel, W.: The relativistic Boltzman equation "General Relativity". Papers in honour of J. L. Synge, Oxford: Clarendon Press 1972

9. Choquet-Bruhat, Y., Geroch, R.: Global aspects of the Cauchy problem in general relativity, Commun. math. Phys. 14, 329 (1971)

Yvonne Choquet-Bruhat

Département Mécanique

Université Paris 6

F-75 Paris, France 